\title{
The influence of maternal pregnancy glucose concentrations on associations between a fetal imprinted gene allele score and offspring size at birth
}

\author{
Clive J. Petry ${ }^{1 *} \mathbb{D}$, Ken K. Ong ${ }^{1,2,3}$, leuan A. Hughes ${ }^{1}$, Carlo L. Acerini ${ }^{1}$ and David B. Dunger ${ }^{1,3}$
}

\begin{abstract}
Objective: Previously we found that certain fetal imprinted genes represented as an allele score are associated with maternal pregnancy glucose concentrations. Recently it was reported that fetal polymorphisms with strong associations with birth weight tend to mediate these independently of increases in maternal pregnancy glucose concentrations. We therefore investigated whether potential associations between the fetal allele score and birth weight were related to maternal glucose concentrations in the Cambridge Baby Growth Study.

Results: The fetal imprinted gene allele score was positively associated with birth weight ( $\beta=63(17-109) \mathrm{g} / \mathrm{risk}$ allele, $\left.\beta^{\prime}=0.113, p=7.6 \times 10^{-3}, n=405\right)$. This association was partially attenuated by adjusting for maternal glucose concentrations ( $\beta=50$ (4-95) g/risk allele, $\left.\beta^{\prime}=0.089, p=0.03, n=405\right)$. The allele score was also positively associated with risk of being large for gestational age at birth (odds ratio $1.60(1.19-2.15)$ per risk allele, $p=2.1 \times 10^{-3}, n=660$ ) and negatively associated with risk of being small for gestational age at birth (odds ratio 0.65 (0.44-0.96) per risk allele, $p=0.03, n=660$ ). The large for gestational age at birth association was also partially attenuated by maternal glucose concentrations. These results suggest that associations between the fetal imprinted gene allele score and size at birth are mediated through both glucose-dependent and glucose-independent mechanisms.
\end{abstract}

Keywords: Gestational diabetes, Pregnancy, Growth, Development, Cohort study

\section{Introduction}

Fetal exposure to glucose is thought to be one of the principal stimulators of growth in utero [1-3]. It is believed that glucose-stimulated fetal insulin secretion stimulates growth whether or not pregnancies are affected by diabetes [4]. Indeed particularly prior to the third trimester of pregnancy when its glucoregulatory function develops, the principal roles of fetal insulin are both mitogenic and anabolic, such as enhancing the growth of white adipocytes and stimulating triglyceride production and deposition in them [5].

\footnotetext{
*Correspondence: cjp1002@cam.ac.uk

1 Department of Paediatrics, Addenbrooke's Hospital, University of Cambridge, Hills Road, Box 116, Cambridge CB2 0QQ, UK

Full list of author information is available at the end of the article
}

Genetics can have roles in regulating both maternal glucose concentrations in pregnancy and fetal growth expressed as offspring birth weight. We recently reported associations between fetal imprinted genes represented as an allele score and both gestational diabetes and maternal glucose concentrations in late pregnancy [6]. In a birth weight-related genome wide association study (GWAS) Beaumont et al. [7] recently reported certain maternal polymorphic variants that were associated with offspring birth weights where the equivalent fetal variants were not. In contrast certain other variants were associated with offspring birth weight in both the maternal and fetal genes. Interestingly all these maternal variants, where the fetal equivalent were not associated with offspring birth weights, were also associated with maternal glucose concentrations in pregnancy [7]. 
A subsequent study found that a gene score constructed using the principal fetal birth weight variants (from [8]) was strongly associated with birth weights independently of effects of maternal glucose concentrations [9]. The authors of this study concluded that for any level of maternal glucose concentration fetal genetics has a major impact on growth but acts predominantly through mechanisms independent of maternal glucose. However none of the fetal variants used to construct the gene score in this study were independently associated with maternal glucose concentrations. In the current study we therefore examined relationships between our fetal imprinted gene allele score and measures of size at birth, in particular investigating whether any allele score associations with size at birth appear to be attenuated by maternal glucose concentrations in pregnancy.

\section{Main text Methods \\ Cambridge baby growth study}

The first phase of the prospective, longitudinal Cambridge Baby Growth Study recruited mothers (and their partners and offspring) attending early pregnancy ultrasound clinics at the Rosie Maternity Hospital, Cambridge, UK between the years 2001-2009 [6, 10]. At around 28 weeks of gestation the mothers underwent a $75 \mathrm{~g}$ oral glucose tolerance test (OGTT) after fasting overnight. Plasma glucose concentrations were measured using a standard glucose oxidase-based procedure on samples collected when fasting and $60 \mathrm{~min}$ after the consumption of the glucose load. Offspring birth weight and gestational age at birth data were collected from hospital notes. Large for gestational age (LGA) at birth was defined as being in the top decile for birth weight adjusted for gestational age. Similarly small for gestational age at birth (SGA) was defined as being in the bottom decile. In this cohort $96.9 \%$ of the offspring were Caucasian, $0.8 \%$ were mixed race, $0.6 \%$ were Afro-Caribbean, $0.8 \%$ were Oriental and $0.9 \%$ were Indo-Asian. The current analysis was restricted to pregnancies where both fasting and 60 min OGTT glucose concentrations were available.

\section{Genotyping and fetal allele score formulation}

Blood or mouth swab samples for DNA extraction were collected from 845 family (mother, father and baby) trios of the 1074 families where maternal OGTT data were available. Genomic DNA was extracted from these samples using an Autopure LS Machine (Qiagen Ltd., Crawley, UK). Allelic transmission to the fetus was imputed from the DNA family trio genotypes $[6,10]$, with the genotyping performed using Kompetitive Allele Specific PCR assays (by LGC Genomics, Hoddesdon, UK). The genotypes that were used in this study were all consistent with Hardy-Weinberg equilibrium ( $p>0.05$ using the $X^{2}$ test) and had a repeat genotyping discordancy rate of $<1.0 \%$. The unweighted fetal allele score was formulated as previously described [6] using the fetal paternally-transmitted INS-IGF2 rs10770125 and rs2585, and maternally-transmitted KCNQ1 rs231841 and rs7929804 alleles.

\section{Statistical analysis}

Associations with offspring birth weight were tested using linear regression, adjusted for established co-variates. Associations with LGA and SGA were tested using both logistic and linear regression. $\mathrm{P}<0.05$ was considered statistically significant throughout. Data are mean ( $95 \%$ confidence interval) unless stated otherwise.

\section{Results}

\section{Associations with offspring birth weight}

The covariates in the regression models (gestational age at birth, sex, parity, maternal pre-pregnancy body mass index, pregnancy weight gain and maternal smoking during pregnancy) explained $31.5 \%$ of the variance in the offspring birth weights by themselves. OGTT fasting glucose concentrations were significantly associated with offspring birth weights when added to the model $\left(\beta=0.16(0.09-0.23) \mathrm{g} \mathrm{l} / \mathrm{mmol}, \beta^{\prime}=0.150, \mathrm{p}=1.5 \times 10^{-5}\right.$, $\mathrm{n}=609$ ), as were OGTT $60 \mathrm{~min}$ glucose concentrations $\left(\beta=0.05(0.03,0.07) \mathrm{gl} / \mathrm{mmol}, \beta^{\prime}=0.161, \mathrm{p}=4.4 \times 10^{-6}\right.$, $\mathrm{n}=602)$. The fetal allele score was also positively associated with birth weight $(\beta=63$ (17-109) g/risk allele, $\beta^{\prime}=0.113, p=7.6 \times 10^{-3}, n=405$ ) (Fig. 1). This association was partially attenuated when adjusting for the OGTT fasting and $60 \mathrm{~min}$ glucose concentrations $(\beta=50$ (4-95) g/risk allele, $\beta^{\prime}=0.089, \mathrm{p}=0.03, \mathrm{n}=405$ ), shown by the flatter slope of the predicted line of best fit of the model (Fig. 1).

\section{Associations with being large or small for gestational age at birth}

The fetal allele score was positively associated with risk of being LGA [odds ratio (OR) $1.60(1.19-2.15)$ per risk allele, $\mathrm{p}=2.1 \times 10^{-3}$ ] (Fig. 2a). The association was only partially attenuated by adjusting for OGTT fasting and 60 min glucose concentrations [OR 1.47 (1.09-1.98) per risk allele, $\mathrm{p}=0.01)$ ]. The fetal allele score was also negatively associated with risk of being SGA [OR 0.65 (0.440.96 ) per risk allele, $\mathrm{p}=0.03$ ] (Fig. 2b). The association was not attenuated when adjusting for OGTT fasting and 60 min glucose concentrations [OR $0.67(0.49-0.99)$ per risk allele, $\mathrm{p}=0.04]$. 


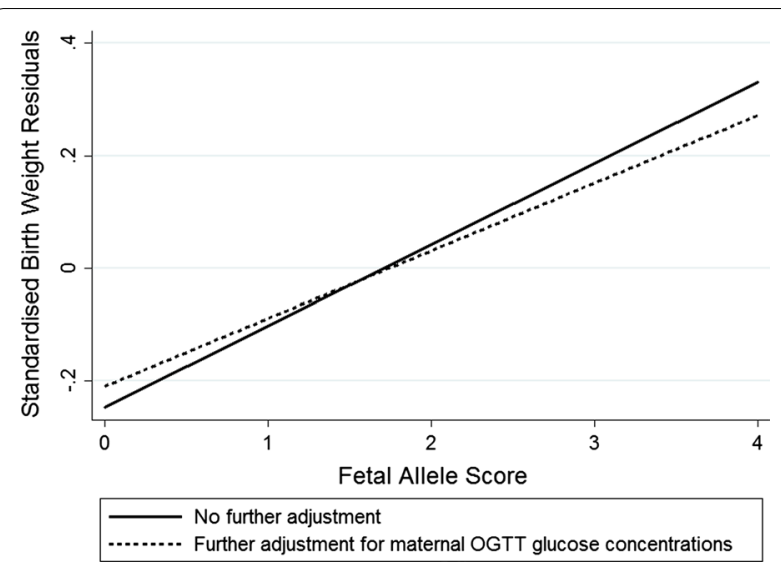

Fig. 1 Linear fit prediction plots showing the relationships between the fetal allele score and standardised birth weight residuals (after adjustment for gestational age at birth, sex, parity, maternal pre-pregnancy body mass index, pregnancy weight gain and maternal smoking during pregnancy). The plots are shown with and without further adjustment for maternal OGTT glucose concentrations, this adjustment flattening the slope

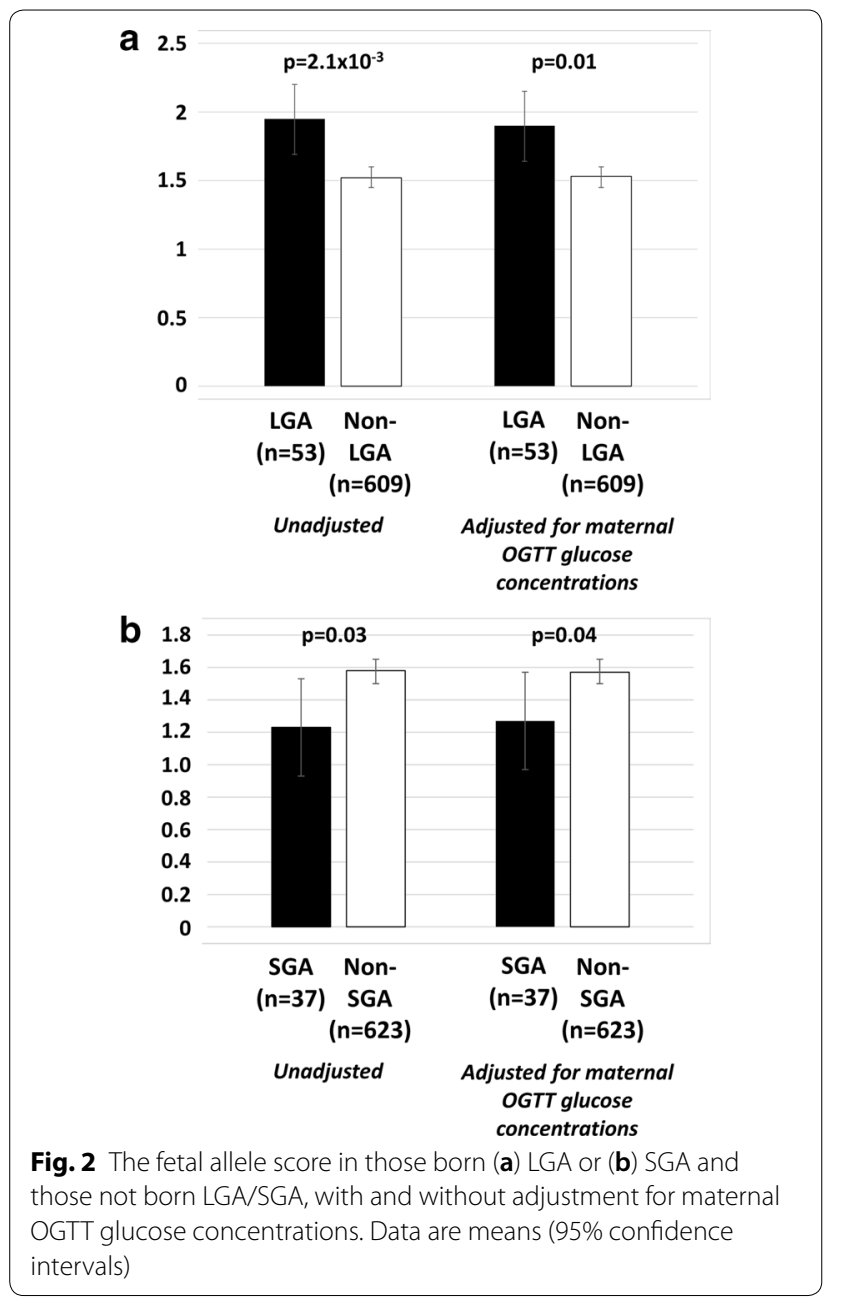

\section{Discussion}

In this study the fetal imprinted gene allele score that we had previously shown to be associated with both maternal glucose concentrations and gestational diabetes risk [6], was additionally associated with offspring birth weight and risk of being LGA or SGA. This is perhaps not surprising given the enrichment of imprinted gene regions identified in the largest birth weightrelated GWAS using fetal genotypes [8]. The effect sizes were partially attenuated when the associations were further adjusted for week 28 OGTT fasting and $60 \mathrm{~min}$ maternal glucose concentrations, suggesting that the link between the fetal allele score and birth weight is mediated through both glucose-dependent and glucose independent mechanisms. These findings are therefore somewhat inconsistent with the associations reported by Hughes et al. [9] where their fetal gene score was associated with birth weight completely independently of maternal glucose concentrations. The difference in the results of the two studies is probably due to the way that the two fetal gene scores were formulated: that used by Hughes et al. [9] being put together from fetal polymorphisms strongly associated with birth weights and our allele score being established using fetal alleles found to be associated with maternal glucose concentrations. Whilst increased maternal glucose concentrations are known to lead to increased birth weights [4], glucose-independent pathways upregulated in gestational diabetes that could potentially affect offspring birth weight include increased placental transport of both fatty acids and certain amino acids $[11,12]$.

The main strengths of this study are its prospective nature and the use of a novel fetal imprinted gene allele score that we found to be robustly associated with maternal glucose concentrations, even to genome wide significance levels by meta-analysis of three different birth cohorts [6]. Its conclusion is that some of the principal fetal imprinted gene variants that are associated with maternal glucose concentrations in late pregnancy in our studies (fetal paternally-transmitted INS-IGF2 rs10770125 and rs2585, and maternallytransmitted KCNQ1 rs231841 and rs7929804 [6, 10]), are collectively associated with birth weight through both maternal glucose-dependent and glucose-independent mechanisms.

\section{Limitations}

- The study has a modest (and variable) sample size which restricted its statistical power.

- The lack of validation of the associations in additional cohorts. 
- The lack of adjustment of the p-values for multiple testing.

\section{Abbreviations}

GWAS: genome wide association study; LGA: large for gestational age; OGTT: oral glucose tolerance test; OR: odds ratio; SGA: small for gestational age.

\section{Authors' contributions}

CP designed and analysed the data for this specific study and drafted the manuscript with DD. KO, IH, CA and DD designed, established and oversee the Cambridge Baby Growth Study. All authors commented on the draft manuscript. All authors read and approved the final manuscript.

\section{Author details}

'Department of Paediatrics, Addenbrooke's Hospital, University of Cambridge, Hills Road, Box 116, Cambridge CB2 0QQ, UK. ${ }^{2}$ Medical Research Council Epidemiology Unit, University of Cambridge, Cambridge CB2 0QQ, UK. ${ }^{3}$ The Institute of Metabolic Science, University of Cambridge, Cambridge CB2 OQQ, UK.

\section{Acknowledgements}

The authors acknowledge the excellent technical assistance in the laboratory for this project that was provided by Dianne Wingate, Rachel Seear, Katrin Mooslehner and Radka Platte. The authors would like to thank all the families that took part in the Cambridge Baby Growth Study, and acknowledge the crucial role played by the research nurses especially Suzanne Smith, Ann-Marie Wardell and Karen Forbes, staff at the Addenbrooke's Wellcome Trust Clinical Research Facility, and midwives at the Rosie Maternity Hospital in collecting data for this study.

\section{Competing interests}

The authors declare that they have no competing interests.

\section{Availability of data and materials}

The datasets generated and/or analysed during the current study are available in the Apollo repository (https://doi.org/10.17863/CAM.31534).

\section{Consent for publication}

Not applicable.

\section{Ethics approval and consent to participate}

The Cambridge Baby Growth Study was approved by the Addenbrooke's Hospital Local Ethics Committee, Cambridge, UK. Written informed consent was obtained from the parents, including consent for inclusion of their babies in the study.

\section{Funding}

This work was supported by the Evelyn Trust (grant number EW9035322); Diabetes UK (Grant Number 11/0004241); the Wellbeing of Women (the Royal College of Obstetricians and Gynaecologists, UK) (Grant Number RG1644); the Medical Research Council (Grant Numbers G1001995, 7500001180); European Union Framework 5 (Grant Number QLK4-1999-01422); the Mothercare Charitable Foundation (Grant Number RG54608); Newlife Foundation for Disabled Children (Grant Number 07/20); the World Cancer Research Fund International
(Grant Number 2004/03); and the National Institute for Health Research Cambridge Biomedical Research Centre. KO is supported by the Medical Research Council (Unit Programme Number: MC_UU_12015/2). None of the funding bodies influenced the design of the study or the collection, analysis or the interpretation of the data used in this study. Neither did they influence the writing of this manuscript.

\section{Publisher's Note}

Springer Nature remains neutral with regard to jurisdictional claims in published maps and institutional affiliations.

Received: 19 October 2018 Accepted: 14 November 2018

Published online: 19 November 2018

\section{References}

1. Parretti E, Mecacci F, Papini M, Cioni R, Carignani L, Mignosa M, et al. Thirdtrimester maternal glucose levels from diurnal profiles in nondiabetic pregnancies: correlation with sonographic parameters of fetal growth. Diabetes Care. 2001;24:1319-23.

2. Walsh JM, Mahony R, Byrne J, Foley M, McAuliffe FM. The association of maternal and fetal glucose homeostasis with fetal adiposity and birthweight. Eur J Obstet Gynecol Reprod Biol. 2011;159:338-41.

3. Sung JF, Kogut EA, Lee HC, Mannan JL, Navabi K, Taslimi MM, et al. Correlation of continuous glucose monitoring profiles with pregnancy outcomes in nondiabetic women. Am J Perinatol. 2015;32:461-8.

4. Hay WW Jr. Recent observations on the regulation of fetal metabolism by glucose. J Physiol. 2006;572:17-24

5. Desoye G, van Poppel M. The feto-placental dialogue and diabesity. Best Pract Res Clin Obstet Gynaecol. 2015;29:15-23.

6. Petry CJ, Mooslehner K, Prentice P, Hayes MG, Nodzenski M, Scholtens $\mathrm{DM}$, et al. Associations between a fetal imprinted gene allele score and late pregnancy maternal glucose concentrations. Diabetes Metab. 2017:43:323-31.

7. Beaumont RN, Warrington NM, Cavadino A, Tyrrell J, Nodzenski M, Horikoshi $\mathrm{M}$, et al. Genome-wide association study of offspring birth weight in 86577 women identifies five novel loci and highlights maternal genetic effects that are independent of fetal genetics. Hum Mol Genet. 2018:27:742-56.

8. Horikoshi M, Beaumont RN, Day FR, Warrington NM, Kooijman MN, Fernandez-Tajes J, et al. Genome-wide associations for birth weight and correlations with adult disease. Nature. 2016;538:248-52.

9. Hughes AE, Nodzenski M, Beaumont RN, Talbot O, Shields BM, Scholtens $D M$, et al. Fetal genotype and maternal glucose have independent and additive effects on birth weight. Diabetes. 2018:67:1024-9.

10. Petry CJ, Seear RV, Wingate DL, Manico L, Acerini CL, Ong KK, et al. Associations between paternally transmitted fetal IGF2 variants and maternal circulating glucose concentrations in pregnancy. Diabetes. 2011;60:3090-6.

11. Ruiz-Palacios M, Ruiz-Alcaraz AJ, Sanchez-Campillo M, Larqué E. Role of Insulin in placental transport of nutrients in gestational diabetes mellitus. Ann Nutr Metab. 2017;70:16-25.

12. Gallo LA, Barrett HL, Dekker Nitert M. Placental transport and metabolism of energy substrates in maternal obesity and diabetes. Placenta. 2017;54:59-67. 